\title{
Mutual coupling and electromagnetic diffraction influence on GPS microstrip antenna arrays
}

\author{
Ezequiel Alejandro Marranghelli \\ Instituto LEICI \\ CONICET - Univ. Nac. de La Plata \\ La Plata, Buenos Aires \\ Email: emarranghelli@gmail.com
}

\author{
G. Ramón Lopez La Valle \\ Instituto LEICI \\ Univ. Nac. de La Plata - CONEAU \\ La Plata, Buenos Aires \\ Email: lopezlavalle@ing.unlp.edu.ar
}

\author{
Pedro Agustín Roncagliolo \\ Instituto LEICI \\ Univ. Nac. de La Plata - CONEAU \\ La Plata, Buenos Aires \\ Email: agustinr@ing.unlp.edu.ar
}

\begin{abstract}
We analize the performance of a microstrip antenna array considering mutual coupling (MC) and diffraction phenomenon (DF). These effects are the reason of distortions on the individual radiation patterns of each antenna when they are placed together. A four element plane array capable of receiving GPS signals at L1 band is analized. The effects of MC and DF are quantified through numerical simulations to compare the advantages of knowing the implemented beamforming based on the characterized distorted patterns. Results prove that, for a few elements array, precise knowledge of each individual radiation pattern in the array is essential for achieving good performance on interferences mitigation. Finally, first measures of a built prototype are shown and they fit properly with simulations.
\end{abstract}

Index Terms-Antenna Array, Mutual Coupling, Difraction, Digital Beamforming, Interference Mitigation.

\section{INTRODUCCIÓN}

Los Sistemas de Navegación Global por Satélite (GNSS) permiten determinar la posición y velocidad de un receptor capaz de sincronizarse con las señales transmitidas por sus satélites. Los GNSS poseen numerosas aplicaciones en diferentes ámbitos, sin embargo en los últimos años su uso civil ha crecido notablemente. De los GNSS existentes en la actualidad, el más difundido y utilizado es el Sistema de Posicionamiento Global (GPS), que se encuentra completamente operativo desde 1994.

Las señales GNSS son susceptibles a interferencias porque sus niveles de potencia son muy bajos debido a que provienen de satélites que se encuentran a una gran distancia de la Tierra. Una técnica ampliamente utilizada para mitigar el efecto de las interferencias consiste en emplear un arreglo de antenas para conformar un diagrama de radiación con un nulo en la dirección de la que proviene la señal interferente [1], [2].

En los arreglos de antenas existen dos fenómenos conocidos como acoplamiento mutuo (MC) y efecto de difracción (DF), los cuales modifican inherentemente el diagrama de radiación de cada antena dispuesta en el arreglo si se lo compara con el que se obtiene cuando la antena se encuentra aislada. En consecuencia, si para calcular el diagrama de radiación conformado del arreglo se utilizan los diagramas individuales de las antenas aisladas, es decir sin tener en cuenta los efectos acoplamiento y difracción, los resultados obtenidos pueden diferir notablemente de los buscados. Estos efectos han sido ámpliamente estudiados en la literatura para el caso de arreglos formados por dipolos [3]. No obstante, para las frecuencias de los GNSS las antenas de microtira ofrecen una solución más viable por su bajo costo, robustez y bajo perfil. En este tipo de antenas los efectos de difracción son más apreciables ya que ocurren mayormente sobre los bordes abruptos de sus elementos conductores [4], [5].

El diagrama de radiación de cada elemento de un arreglo de antenas puede calcularse a partir del Método de Excitación Unitaria del Patrón del Elemento Activo [6]. Diversos autores han empleado estas ideas y han corroborado que las técnicas de compensación del MC no son suficientes si se consideran diagramas de radiación de elementos aislados en lugar de considerar elementos activos [7], [8]. Algunas técnicas para compensar o predecir el MC entre elementos radiantes están recopiladas en [9].

El objetivo de este trabajo es comparar por métodos numéricos el desempeño de un arreglo de antenas de microtira empleando el método de excitación unitaria del elemento activo con la muy conocida técnica de multiplicación de un diagrama de radiación ideal aislado por un factor de arreglo [10]. Debido a que normalmente las antenas de un receptor de GPS son colocadas sobre vehículos o soportes fijos, es habitual que las mismas se encuentren montadas sobre estructuras metálicas. Por ello, se considera también la influencia de un soporte conductor sobre el que se disponen las antenas del arreglo.

El resto del trabajo está organizado de la siguiente forma. En la Sección 2 se describe cómo se conforma el diagrama de radiación de un arreglo de antenas en base a los diagramas de las antenas individuales y se analizan los efectos de acoplamiento y difracción involucrados. En la Sección 3 se presenta el diseño de un arreglo plano de cuatro antenas aplicado a la recepción de señales de GPS. La Sección 4 está destinada a presentar los resultados de las simulaciones realizadas al arreglo propuesto. En la Sección 5 se aplica la conformación de haz y se comparan los resultados obtenidos para los casos analizados. En la Sección 6 se presentan las mediciones realizadas al arreglo construido. Finalmente, en la Sección 7 se exponen las conclusiones obtenidas. 


\section{PATRÓN DE RADIACIÓN DE UN ARREGLO}

El diagrama de radiación de un arreglo de $N$ antenas puede ser calculado exactamente empleando el Método de Excitación-Unitaria del Elemento Activo como

$$
F(\theta, \phi)=\sum_{n=1}^{N} a_{n} . g_{n}(\theta, \phi)
$$

donde $a_{n}$ es el n-ésimo coeficiente del vector de coeficientes a del arreglo (determinado por el algoritmo de conformación de haz utilizado) y $g_{n}$ es el n-ésimo diagrama de excitaciónunitaria del elemento activo. Se llama diagrama de radiación activo de excitación unitaria al diagrama de radiación generado por una antena que es excitada por una fuente de tensión unitaria, a la frecuencia de operación, y que se encuentra en presencia del resto de los elementos del arreglo cargados con impedancias de carga que coinciden con la impedancia equivalente de sus correspondientes generadores.

La suposición típica al momento de calcular el diagrama de radiación de una antena es considerar que la misma se encuentra aislada e inmersa en un medio lineal, isotrópico, homogéneo y de bajas pérdidas (LIH). Al trabajar con arreglos donde la proximidad entre los elementos es del orden de la longitud de onda, este modelo ideal deja de ser apropiado. Los dieléctricos con distintas propiedades electromagnéticas y los materiales conductores alteran el medio LIH y, por lo tanto, los campos generados por cada una de las antenas se reflejan, se refractan y se difractan en distintas direcciones. Además existe la difracción de campo cercano, cuyo comportamiento es igualmente lineal, que redirecciona aquellos rayos que inciden sobre bordes abruptos y esquinas de elementos conductores. Estos rayos difractados se desvían en infinitas direcciones combinándose con las ondas EM de rayo directo generadas por la propia antena fuente.

Por otro lado, la energía que una antena irradia es captada en cierta proporción por sus elementos vecinos y, dado que se trata de elementos igualmente resonantes, parte de esa energía volverá a ser irradiada, pero esta vez por un nuevo agente. Este comportamiento se conoce como MC entre antenas y puede calcularse como una combinación lineal entre diagramas de radiación "aislados" $g_{m}^{\prime}(\theta, \phi)$ de la siguiente forma

$$
g_{n}(\theta, \phi)=\sum_{m=1}^{N} c_{n m} \cdot g_{m}^{\prime}(\theta, \phi)
$$

donde $g_{n}(\theta, \phi)$ es el diagrama de radiación activo de la antena n-ésima y $g_{m}^{\prime}(\theta, \phi)$ es el diagrama de radiación aislado de la antena m-ésima. En este trabajo se ha tomado como definición de antena aislada a la propia antena ubicada en el entorno en el que realmente se encuentra pero sin tomar en cuenta el MC entre elementos. Es decir

$$
g_{m}^{\prime}(\theta, \phi)=E_{m}^{i}(\theta, \phi)+E_{m}^{r}(\theta, \phi)+E_{m}^{d}(\theta, \phi)
$$

siendo $E_{m}^{i}(\theta, \phi)$ y $E_{m}^{r}(\theta, \phi)$ los campos directos y reflejados del elemento radiante respectivamente y $E_{m}^{d}(\theta, \phi)$ el campo

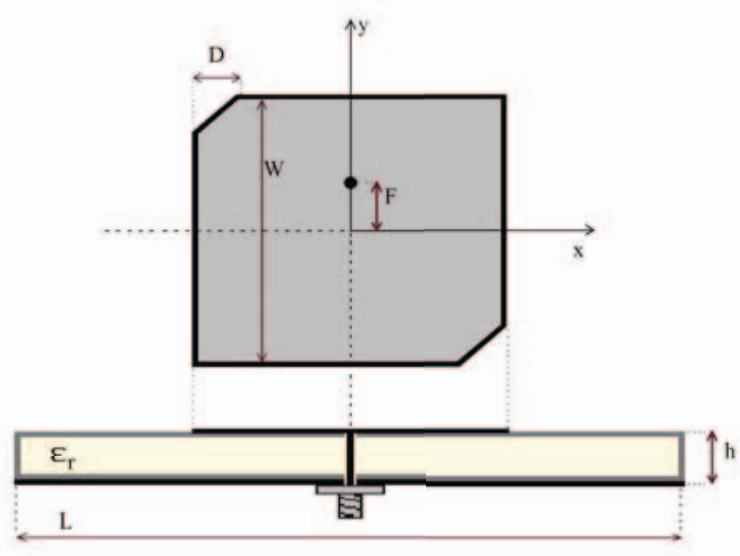

Figure 1. Antena propuesta sintonizada en $1575 \mathrm{MHz}$.

difractado total que se obtiene a partir de la suma de todos los rayos difractados en distintos puntos de bordes y esquinas de los materiales conductores incluidos en el modelo. Teniendo en cuenta lo mencionado anteriormente el diagrama de radiación del arreglo queda expresado como

$$
F(\theta, \phi)=\sum_{n=1}^{N} a_{n} \cdot\left(\sum_{m=1}^{N} c_{n m} \cdot g_{m}^{\prime}(\theta, \phi)\right)
$$

En condiciones de perfecta adaptación de todos los elementos resonantes del arreglo, el MC sería fácilmente medible ya que en este caso podríamos asegurar que la energía reirradiada por los mismos es igual a la energía disipada en la impedancia de carga (suponiendo máxima eficiencia). Bajo este contexto es posible conocer y descontar el efecto producido por MC para así obtener los diagramas de radiación de antenas aisladas.

\section{DISEÑO DEL ARREGLO}

Los receptores GNSS normalmente emplean antenas de microtira ya que éstas se caracterizan por ser hemiesféricas, lo cual es importante para maximizar la visibilidad de satélites. Por otra parte, las antenas de microtira tienen la capacidad de polarizarse en forma circular, son de costo reducido, mecánicamente robustas y de bajo perfil. En particular, en este trabajo se optó por utilizar antenas de microtira con patch cuadrado y esquinas truncadas para lograr polarización circular derecha [11]. Las antenas poseen alimentación simple tipo coaxial y utilizan un sustrato de material RT/duroid 6002 [12]. Cabe destacar que la antena propuesta se encuentra adaptada para la banda L1 tanto de GPS como del sistema ruso GLONASS, lo cual le brinda mayor versatilidad al diseño. En la Fig. 1 se presenta el modelo de la antena con sus parámetros más relevantes indicados en la imagen.

Para implementar el arreglo se dispusieron cuatro antenas alineadas coplanarmente en forma cuadrada y separadas una distancia $d=0.56 \lambda$, como se muestra en la Fig. 2, siendo $\lambda$ la longitud de onda de la frecuencia central de la banda L1 de 


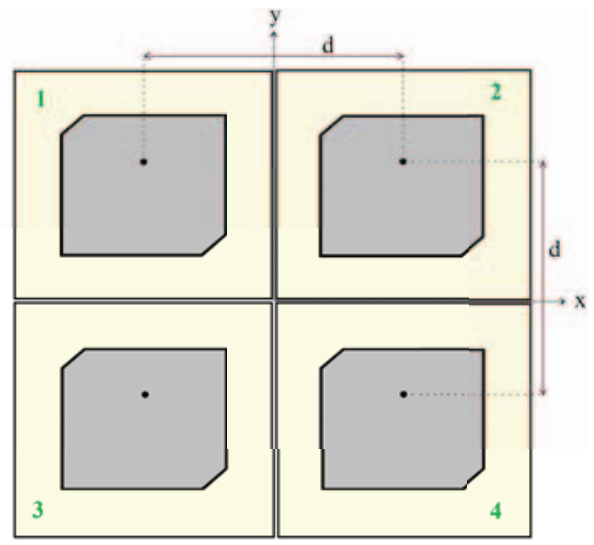

Figure 2. Arreglo $2 \times 2$.

Table I

DIMENSIONES DE LAS ANTENAS.

\begin{tabular}{|c|c|}
\hline Parámetro & Dimensión $[\mathrm{mm}]$ \\
\hline \hline$D$ & 5.2 \\
\hline$F$ & 10.35 \\
\hline$L$ & 100 \\
\hline$W$ & 53.7 \\
\hline$h$ & 1.524 \\
\hline$\varepsilon_{r}$ & 2.94 \\
\hline
\end{tabular}

GPS. La distancia propuesta para la separación entre antenas se debe a la restricción física que imponen las dimensiones de las mismas. Idealmente se utilizaría una separación de $\lambda / 2$ a fin de poder realizar la conformación de haz de manera no ambigua. Con esta separación entre las antenas los efectos de MC y el DF son considerables, lo cual motiva el análisis realizado en este trabajo. Por otro lado, cada antena está construida con un sustrato y plano de tierra propios para reducir la propagación de corrientes superficiales entre ellas.

\section{SIMULACIONES}

Las dimensiones finales de la antena de microtira descrita previamente se obtuvieron por simulación empleando un programa basado en el método de integraciones finitas [13], y se resumen en la Tabla I.

Habiendo simulado la antena de microtira aislada de su entorno (antena ideal) y un conjunto de cuatro antenas idénticas dispuestas en el arreglo propuesto anteriormente, donde sólo la antena i-ésima se encuentra activa, comparamos los resultados. Se ha optado por emplear el sistema de coordenadas Ludwig 3 para facilitar la representación del diagrama de radiación [14]. En las Figs. 3 y 4 se muestran dos cortes de planos ortogonales del diagrama de radiación con polarización circular a derecha (RHCP) de la antena aislada y de cada una de las antenas activas del arreglo. Pueden observarse algunas variaciones en el diagrama de cada antena del arreglo respecto del caso ideal, en particular en ciertas orientaciones existen diferencias en el diagrama de fase de hasta $15^{\circ}$ en la región de interés. Incluso entre diagramas de antenas activas, las diferencias de fase

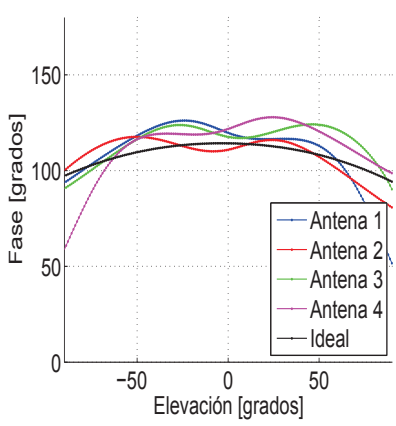

(a)

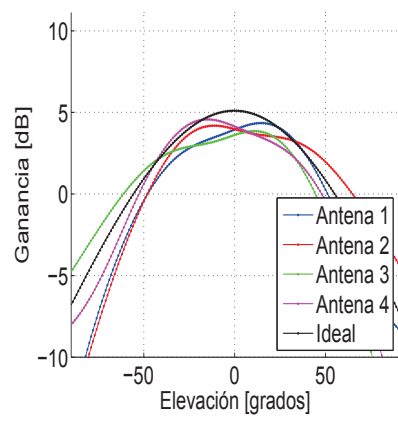

(b)
Figure 3. Patrón de fase (a) y ganancia (b) con RHCP de cada antena activa y la antena aislada en el plano $\phi=0^{\circ}$.

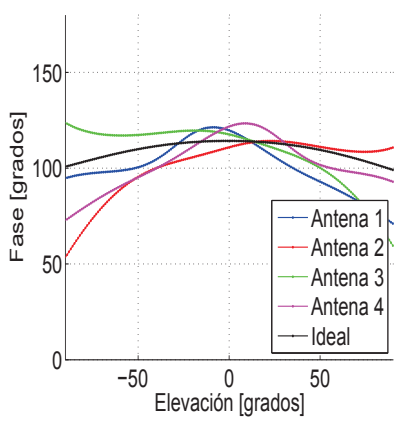

(a)

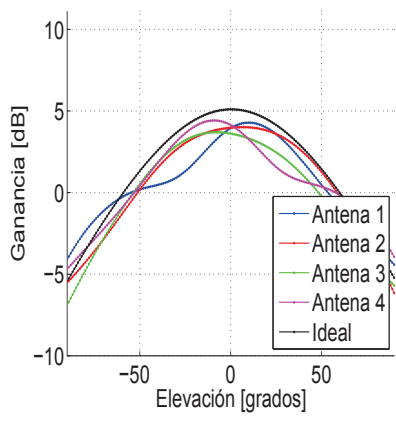

(b)
Figure 4. Patrón de fase (a) y ganancia (b) con RHCP de cada antena activa y la antena aislada en el plano $\phi=90^{\circ}$

pueden ser algo mayores. Puede notarse que las desviaciones respecto al comportamiento ideal de las antenas presentan cierta simetría espacial entre pares de antenas debido a la simetría geométrica del arreglo.

A continuación, se procedió a agregar un soporte formado por un plano metálico a fin de analizar su influencia sobre los diagramas de las antenas del arreglo para distintas dimensiones. En estas condiciones es esperable que se observen interferencias causadas por reflexión y difracción sobre el plano conductor, las cuales pueden alterar la forma en que las antenas se acoplan. En las Figs. 5 y 6 se comparan los mismos cortes presentados anteriormente del diagrama de radiación con polarización circular a derecha (RHCP). Estos gráficos permiten comparar el desempeño de la antena aislada con la antena activa $\mathrm{N}^{\mathrm{o}} 1$ en el arreglo sin y con soporte conductor de dimensiones $22 \times 22$ y $60 \times 60 \mathrm{~cm}^{2}$, centrado en el origen de coordenadas del arreglo y en contacto con los planos de tierra de cada antena individual. Los resultados obtenidos muestran que los diagramas se ven distorsionados, con aparición de máximos y mínimos de interferencia en distintas direcciones, tanto para la ganancia como para la fase.

\section{CONFORMACIÓN DEL HAZ}

Teniendo en cuenta los resultados presentados en la sección anterior, con el objetivo de conformar el haz de radiación total 


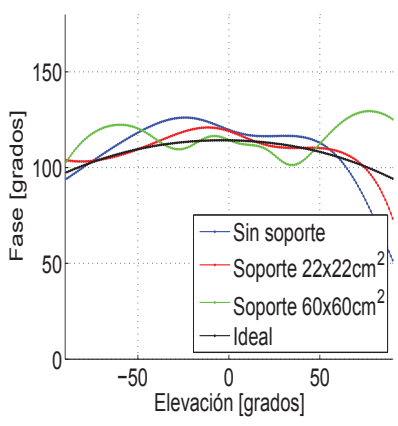

(a)

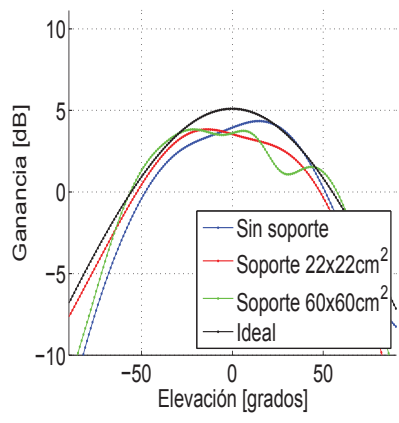

(b)
Figure 5. Patrón de fase (a) y ganancia (b) con RHCP de la antena antena activa $\mathrm{N}^{\mathrm{o}} 1$ para tres esquemas distintos y la antena ideal en el plano $\phi=0^{\circ}$.

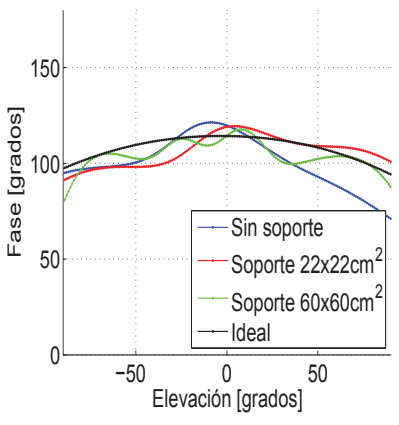

(a)

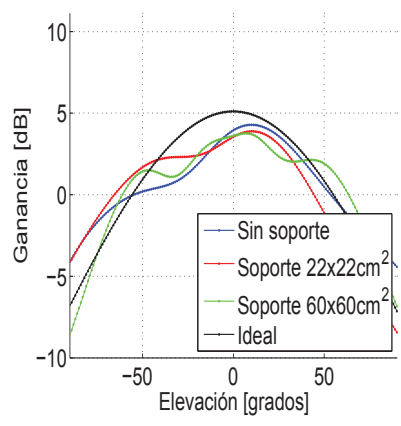

(b)
Figure 6. Patrón de fase (a) y ganancia (b) con RHCP de la antena antena activa $\mathrm{N}^{\circ} 1$ para tres esquemas distintos y la antena ideal en el plano $\phi=90^{\circ}$.

del arreglo con soporte de $60 \times 60 \mathrm{~cm}^{2}$ se analizaron tres casos. En primer lugar se evaluó el diagrama de radiación que se podría obtener orientando el haz en la dirección de máxima ganancia de los elementos individuales, es decir, utilizando todos los coeficientes del arreglo $a_{n}=1$. Por otro lado, se calculó el diagrama "No compensado", que es el que se obtiene si se emplean los mismos coeficientes que en el caso ideal, pero para los diagramas de las antenas individuales con los efectos de MC y DF. Por último, a partir de los diagramas de antenas activas se obtiene el conjunto de coeficientes del arreglo que generan el diagrama llamado "Compensado". Es decir, se corrigen los valores de los coeficientes a fin de compensar las diferencias de amplitud y fase presentes en los diagramas de antenas activas. En la Fig. 7 se muestran los resultados obtenidos.

En la Fig. 8 se presentan los diagramas ideal, no compensado y compensado cuando se orienta el arreglo en dirección a $\theta=40^{\circ}$ elevación y $\phi=180$ azimuth. En este caso, la conformación del haz ideal se realizó simplemente modificando la fase de los coeficientes $a_{n}$ de forma tal de asignar máxima ganancia en la dirección deseada, y la compensación de forma similar al caso anterior. Puede notarse que la máxima ganancia en el caso ideal se obtiene para una elevación menor a la buscada debido a la forma de los diagramas individuales.

Los resultados obtenidos muestran que las diferencias entre

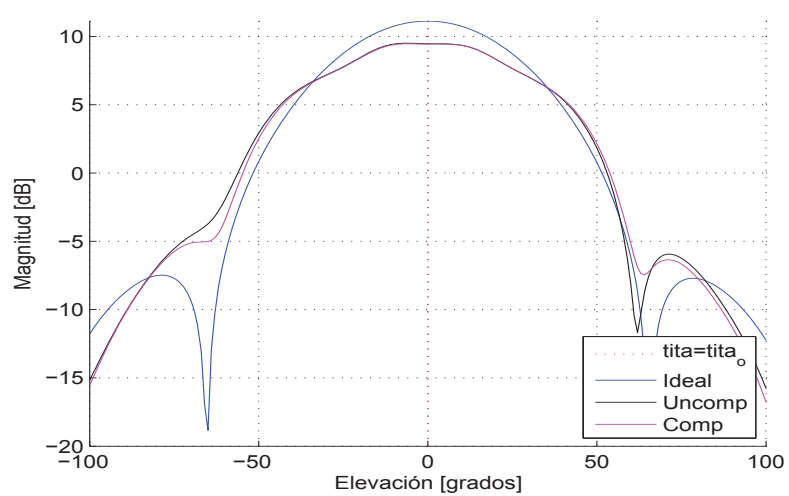

(a)

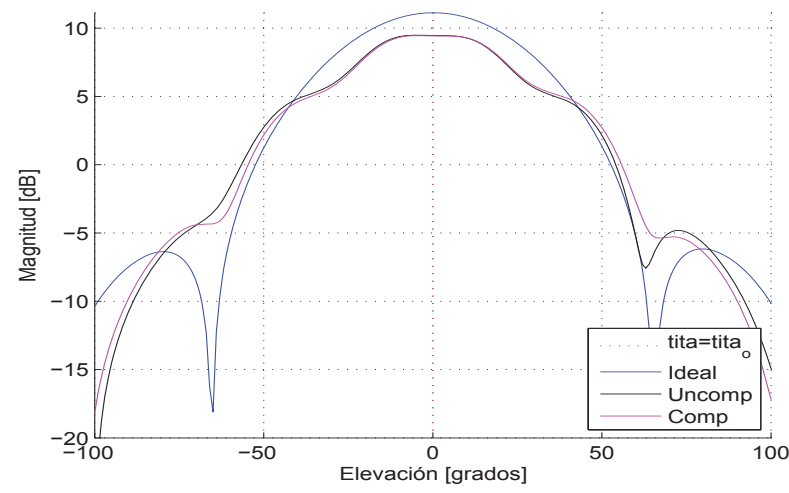

(b)

Figure 7. Patrón de ganancia con RHCP del arreglo de antenas con haz orientado en dirección $\theta=0^{\circ}$. (a) Plano $\phi=0^{\circ} / 180^{\circ}$ y (b) plano $\phi=$ $90^{\circ} / 270^{\circ}$.

el caso ideal y los casos en los que se consideran los efectos de acoplamiento y difracción son relevantes. Sin embargo, para un arreglo plano de cuatro antenas no se aprecian diferencias notables entre el diagrama compensado y el no compensado. Por otro lado, es importante notar que el comportamiento de los nulos difiere notablemente respecto al caso ideal. Esto es de interés cuando se pretende utilizar el arreglo para rechazar interferencias mediante la generación de un nulo en el diagrama conformado. En base a esto, se presentan las Figs. 9 y 10 que ilustran los diagramas ideal, no compensado y compensado cuando se pretende obtener un nulo en dirección a $\left(\theta=30^{\circ}, \phi=180^{\circ}\right)$ y $\left(\theta=40^{\circ}, \phi=50^{\circ}\right)$ respectivamente. Estos resultados dan evidencia de que al considerar antenas ideales dificilmente pueda conseguirse un nulo en la dirección deseada. Para este caso, puede ocurrir que la diferencia entre la ganancia máxima del arreglo y la ganancia en la dirección de la señal interferente alcance a ser de tan solo 6dB. Sin embargo, si se conocen los diagramas activos se podría mejorar la eficacia de atenuación en la dirección deseada, como se puede apreciar claramente en las curvas de los diagramas compensados. 


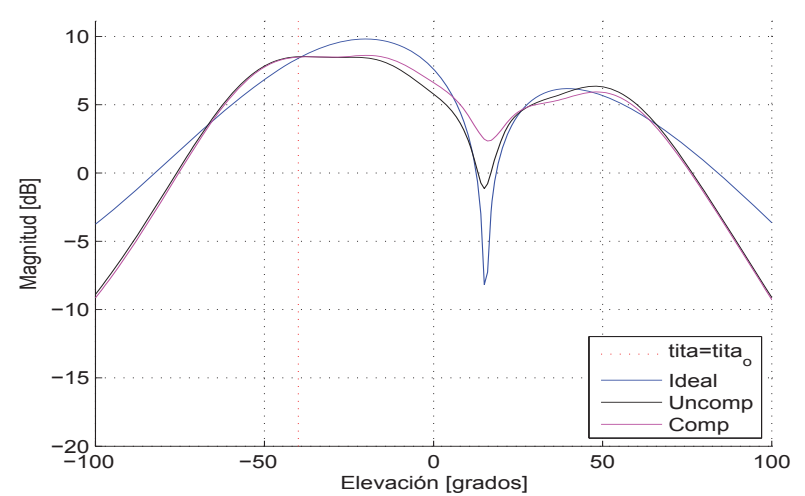

(a)

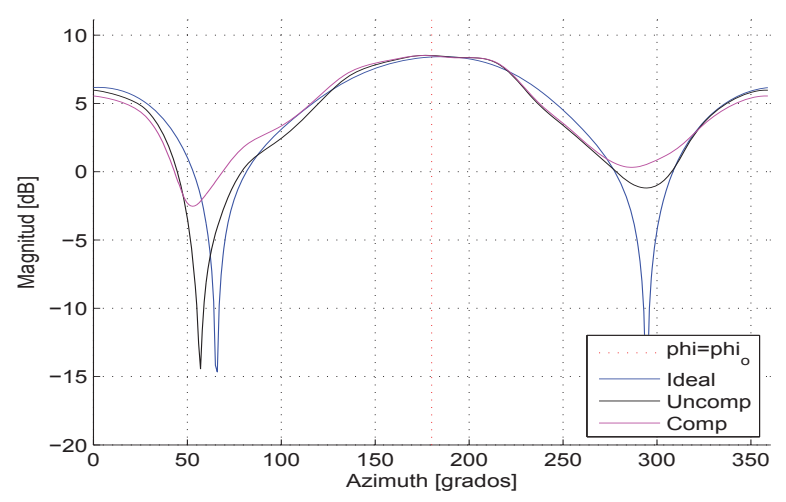

(b)

Figure 8. Patrón de ganancia con RHCP del arreglo de antenas con haz orientado en $\left(\theta=40^{\circ}, \phi=180^{\circ}\right)$. (a) Plano $\phi=180^{\circ}$ y (b) plano $\theta=40^{\circ}$.

\section{IMPLEMENTACIÓN}

Finalmente, se construyó el arreglo propuesto con el objetivo de disponer de un prototipo para realizar pruebas con señales reales y verificar los resultados obtenidos por simulación. Para las antenas se utilizó el diseño descrito en la Sección 3. Como primera medida, para verificar la correspondencia entre el modelo simulado y el construido, se midió la adaptación de cada antena activa utilizando un analizador de redes. Los resultados obtenidos se muestran en la Fig. 11. Puede notarse la buena adaptación de las antenas y la gran semejanza entre los resultados de las mediciones y las simulaciones.

Contando con estos resultados alentadores, se está trabajando actualmente para poder relevar los diagramas de radiación ideales y poder contrastar de forma experimental los resultados presentados en este trabajo y completar la caracterización del arreglo construido.

\section{CONCLUSIONES}

Se analizó el efecto de los fenómenos de difracción y acoplamiento mutuo sobre el diagrama de radiación de un arreglo de antenas de microtira para GPS. En particular, se describió la influencia de estos efectos en el diagrama de

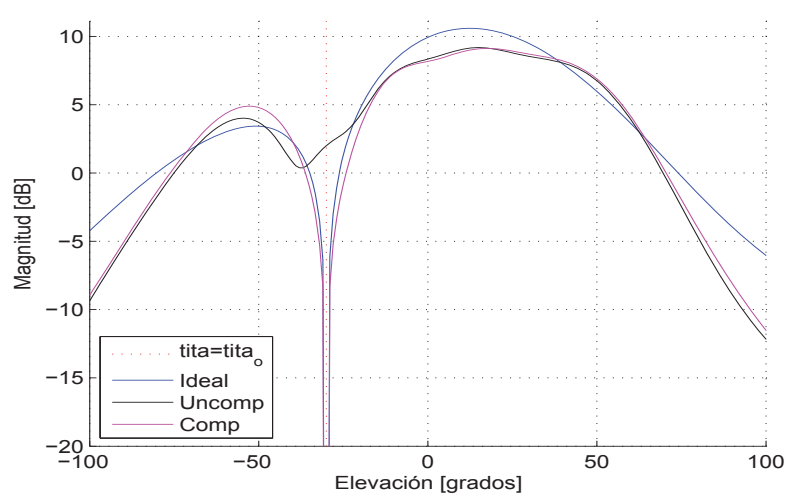

(a)

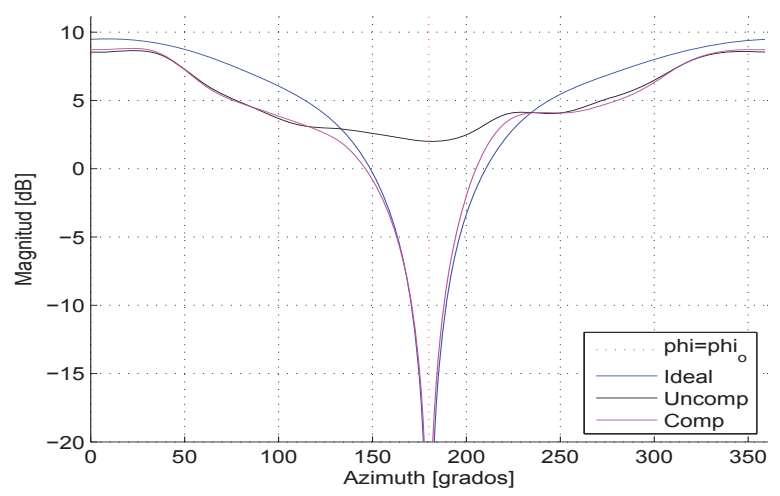

(b)

Figure 9. Patrón de ganancia con RHCP del arreglo de antenas con un nulo en dirección $\left(\theta=30^{\circ}, \phi=180^{\circ}\right)$. (a) Plano $\phi=0^{\circ} / 180^{\circ}$ y (b) plano $\theta=30^{\circ}$.

radiación de las antenas individuales, y a su vez, cómo se ve modificada la forma del diagrama conformado resultante.

Se analizó un arreglo de cuatro antenas, el cual fue caracterizado en forma numérica mediante simulaciones. Las consecuencias del DF y el MC se han visto reflejadas al comparar los diagramas elementales activos con el diagrama ideal de la antena individual. Además se evaluó el desempeño del arreglo montado sobre una superficie metálica, verificándose que la presencia de dicho plano de tierra altera considerablemente la forma de los diagramas de radiación de los elementos del arreglo, dependiendo de su geometría y sus dimensiones. En cuanto al diagrama de radiación conformado, pudo verse que hay notables diferencias entre el diagrama ideal y el real, que se vuelven determinantes en aplicaciones de cancelación de interferencias. Se ha construido un arreglo como el analizado, y se presentaron las primeras mediciones obtenidas que muestran una buena correspondencia entre el modelo simulado y el construido. Actualmente se están realizando medidas experimentales que permitan contrastar los resultados obtenidos en este trabajo.

\section{AGRADECIMIENTOS}

Este trabajo fue financiado por ANPCyT PICT 2011-0909, UNLP Proyecto 11-I-166, CONICET y CIC-PBA. 


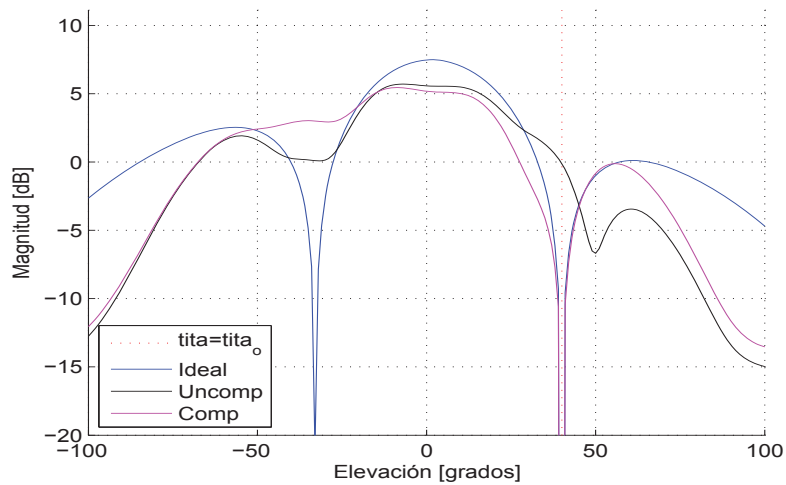

(a)

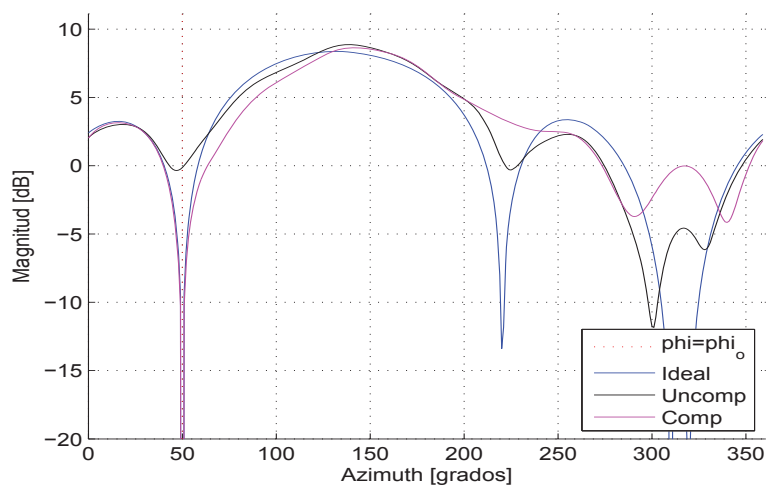

(b)

Figure 10. Patrón de ganancia con RHCP del arreglo de antenas con un nulo en $\left(\theta=40^{\circ}, \phi=50^{\circ}\right)$. (a) Plano $\phi=50^{\circ} / 230^{\circ}$ y (b) plano $\theta=40^{\circ}$.

\section{REFERENCES}

[1] M. G. Amin and W. Sun, "A Novel Interference Suppression Scheme for Global Navigation Satellite Systems Using Antenna Array", IEEE J. Sel. Areas Commun., Vol. 23, No. 5, pp. 999-1012, May 2005.

[2] S. Backen, D. M. Akos, M. L. Nordenvaad, "Post-Processing Dynamic GNSS Antenna Array Calibration and Deterministic Beamforming", Proc. 21 st Int. Tech. Mtg. Satellite Division Inst. Navigation (ION GNSS 2008), Georgia, 2008, pp. 2806-2814.

[3] R. J. Mailloux, Phased Array Antenna Handbook, 2nd edition. Boston: Artech House, 2005.

[4] P. B. S. Kumar and G. S. Ranganath, "Geometrical Theory of Difraction", Pramana, J. Phys., Vol. 37, No. 6, pp. 457-488, December 1991.

[5] C. Balanis, Advanced Engineering Electromagnetics. N. York, USA: J. Wiley \& Sons, 1989.

[6] D. Kelley and W. Stutzman, "Array antenna pattern modeling methods that include mutual coupling effects", IEEE Trans. Antennas Propag., Vol. 41, No. 12, pp. 1625-1632, December 1993.

[7] M. Zamłyński and P. Słobodzian, "Antenna array radiation pattern modelling which includes mutual coupling and diffraction effects", Proc. 6th European Conf. Antennas Propag. (EuCAP 2012), Praga, 2012, pp. 16271631.

[8] M. Zamłyński and P. Słobodzian, "Influence of the aperture edge diffraction effects on the mutual coupling compensation technique in small planar antenna arrays", Int. J. Electron. and Telecommun., Vol. 57, No. 1, pp. 115-120, March 2011

[9] S. Henault and M. M. Antar, "Wideband analysis of mutual coupling compensation methods", Int. J. Antennas Propag., Vol. 2011, pp. 124134, 2011.

[10] H. L. Van Trees, Optimum Array Processing. N. York: J. Wiley \& Sons, 2002.

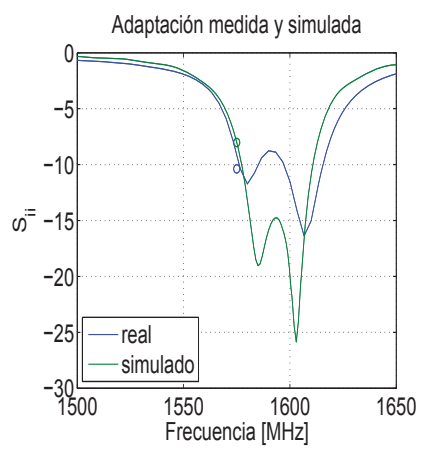

(a)

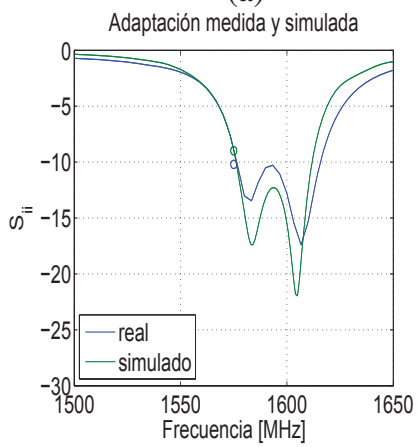

(c)

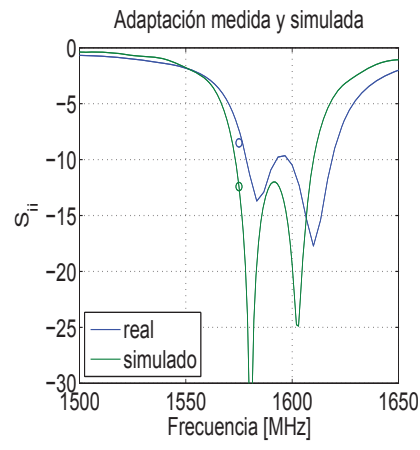

(b)

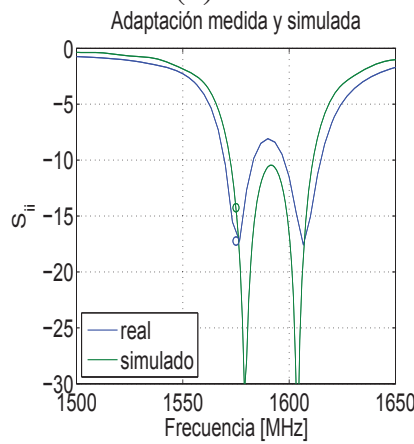

(d)
Figure 11. Adaptación de cada antena simulada y construida (a) Ant. 1, (b) Ant. 2, (c) Ant. 3 y (d) Ant. 4.

[11] C. Balanis, Antenna Theory: Analysis and Design, third edition. N. Jersey, USA: J. Wiley \& Sons, 2005.

[12] Rogers Corporation, "RT/duroid 6002 High Frequency Laminates", Data Sheet, 2011.

[13] T. Weiland, "A discretization method for the solution of Maxwell's equations for six component fields: Electronics and Communication", Int. J. Electron. Comun. (AEÜ), Vol. 31, No. 3, pp. 116-120, 1977.

[14] A. C. Ludwig, "The definition of cross polarization", IEEE Trans. Antennas Propag., Vol 21, No. 1, pp 116-119, January 1973. 